\title{
Mechanisms of Deep Brain Stimulation in Movement Disorders as Revealed by Changes in Stimulus Frequency
}

\author{
Merrill J. Birdno and Warren M. Grill \\ Department of Biomedical Engineering, Duke University, Durham, NC 27708-0281
}

Summary: Deep brain stimulation (DBS) is an established treatment for symptoms in movement disorders and is under investigation for symptom management in persons with psychiatric disorders and epilepsy. Nevertheless, there remains disagreement regarding the physiological mechanisms responsible for the actions of DBS, and this lack of understanding impedes both the design of DBS systems for treating novel diseases and the effective tuning of current DBS systems. Currently available data indicate that effective DBS overrides pathological bursts, low frequency oscillations, synchronization, and disrupted firing patterns present in movement disor- ders, and replaces them with more regularized firing. Although it is likely that the specific mechanism(s) by which DBS exerts its effects varies between diseases and target nuclei, the overriding of pathological activity appears to be ubiquitous. This review provides an overview of changes in motor symptoms with changes in DBS frequency and highlights parallels between the changes in motor symptoms and the changes in cellular activity that appear to underlie the motor symptoms. Key Words: Electrical stimulation, high-frequency stimulation, movement disorders, basal ganglia, thalamus, midbrain.

\section{INTRODUCTION}

Chronic electrical stimulation of the subcortical brain, or deep brain stimulation (DBS), is a surgical intervention that treats motor symptoms in several movement disorders, including Parkinson's disease, essential tremor, and dystonia. ${ }^{1-3}$ DBS electrodes are surgically implanted into specific nuclei of the basal ganglia or thalamus; the location of the electrodes depends on the type of movement disorder and the predominant symptoms of the patient. Wires are then tunneled underneath the skin to an implantable pulse generator that is placed in the upper chest, similar to a cardiac pacemaker. Although outcomes vary among patients, remarkable improvements in motor symptoms result from DBS. Patients with Parkinson's disease experience relief of tremor, rigidity (joint stiffness), and bradykinesia/akinesia (slowness of movement/freezing) during DBS; essential tremor patients experience relief of tremor; and dys-

Address correspondence and reprint requests to: Warren Grill, Ph.D., Duke University, Department of Biomedical Engineering, Hudson Hall, Room 136, Box 90281, Durham, NC 27708-0281. E-mail: warren.grill@duke.edu. tonia patients experience relief of both tonic and phasic dystonic movements.

Despite the clinical effectiveness of DBS, the underlying physiological mechanisms of the effects of DBS are unclear ${ }^{4,5}$ and there are compelling reasons to better understand the mechanisms of DBS. First, the tuning of DBS systems could be significantly improved if we understood the mechanisms of DBS. ${ }^{6,7}$ Several weeks after the surgical implantation, a clinician uses a radio-frequency programmer to set the stimulation parameters. Experienced clinicians are generally successful in quickly reducing the number of potentially successful parameter settings and in tuning DBS systems to treat visible motor symptoms that respond quickly to DBS. However, the time course of responses to DBS can be very long for some symptoms (e.g., gait and postural instability in Parkinson's disease), and it is not clear how to tune DBS systems when there may be no overt effects of stimulation (e.g., epilepsy). An understanding of the mechanisms of DBS would allow clinicians to improve DBS system tuning.

Second, an understanding of DBS mechanisms would enable the application of DBS beyond the realm of movement disorders. DBS is being investigated for treat- 

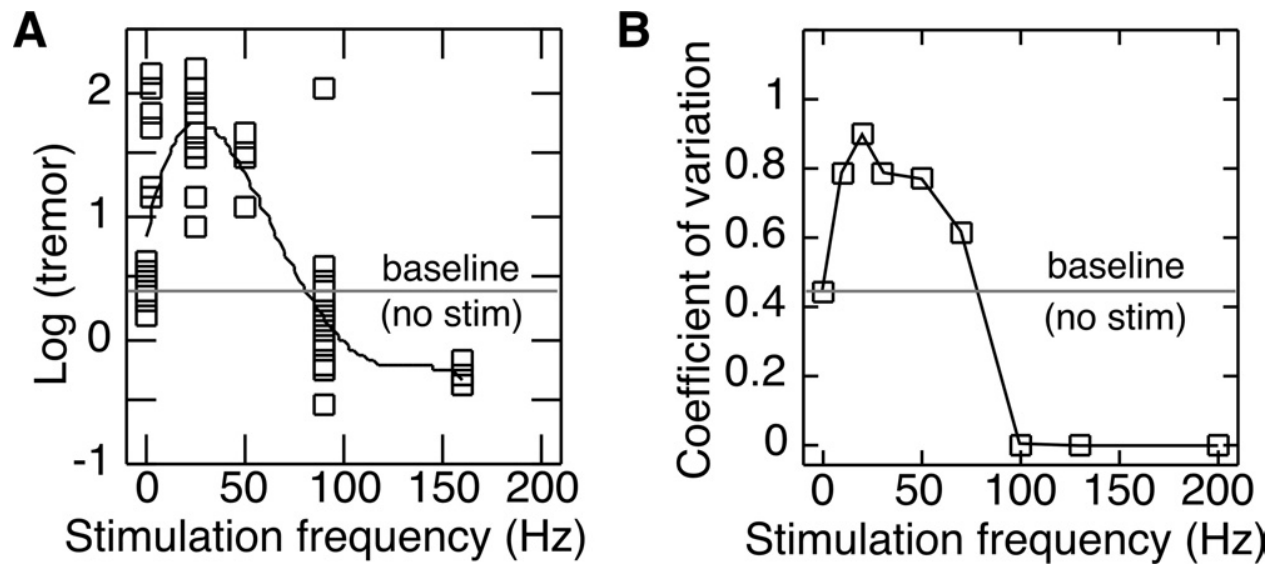

FIG 1. Regularity of model neuron firing parallels tremor as a function of deep brain stimulation frequency. A: Tremor amplitude as a function of deep brain stimulation frequency in the ventral intermediate nucleus of the thalamus in a person with essential tremor. ${ }^{18} \mathrm{~B}$ : Coefficient of variation of the instantaneous firing rates from a bursting thalamocortical model neuron as a function of the frequency of extracellular stimulation. Reproduced with permission from Grill et al. ${ }^{16}$

ment of epilepsy ${ }^{8}$ and psychiatric disorders, ${ }^{9}$ including obsessive-compulsive disorder ${ }^{10}$ and depression. ${ }^{11}$ If we develop a better understanding of the mechanisms of DBS, then we will have a better chance for success in extending DBS treatment to new diseases.

Here, we review the effects of the frequency of DBS on its effectiveness in treating the motor symptoms of movement disorders, as well as the changes in neuronal activity that take place during DBS. Our purpose is not to provide an exhaustive review of all hypothesized mechanisms of DBS, but rather to highlight parallels between the changes in motor symptoms and the changes in cellular activity that appear to underlie the motor symptoms.

\section{DEPENDENCE OF MOTOR SYMPTOMS ON FREQUENCY OF STIMULATION}

The effects of DBS on motor symptoms in Parkinson's disease (PD) and essential tremor are strongly dependent on the stimulation frequency. ${ }^{3,12}$ In subjects with PD, treatment of the three cardinal motor symptoms (i.e., 1, tremor, 2, bradykinesia, and 3, rigidity) improved as the frequency of subthalamic nucleus (STN) DBS was increased and reached a plateau between 130 and 185 Hz. ${ }^{13}$ Similarly, DBS at $130 \mathrm{~Hz}$ led to significant decreases in movement times (i.e., improved bradykinesia) in subjects with PD as compared with stimulation off, ${ }^{14}$ and $130 \mathrm{~Hz}$ stimulation improved scores on the entire motor subset of the Unified Parkinson's Disease Rating Scale (UPDRS). ${ }^{15}$ Conversely, DBS of the STN at $10 \mathrm{~Hz}$ significantly lengthened movement times (i.e., worsened bradykinesia) and significantly worsened UPDRS motor scores. ${ }^{14,15}$ DBS at other low frequencies $(5 \mathrm{~Hz}, 20 \mathrm{~Hz}$, and $45 \mathrm{~Hz}$ ) did not worsen UPDRS motor scores, but was not effective at treating symptoms. ${ }^{15}$

In subjects with ET, tremor suppression also exhibited a strong dependence on thalamic DBS frequency. Stim- ulation at frequencies $<50 \mathrm{~Hz}$ did not suppress tremor, and in some cases it worsened tremor as compared with stimulation off. Conversely, stimulation at frequencies $>90 \mathrm{~Hz}$ suppressed tremor as compared with stimulation off (FIG. 1A). ${ }^{16-18}$ Increasing the stimulation frequency from $90 \mathrm{~Hz}$ to $185 \mathrm{~Hz}$ did not further improve tremor suppression, but rather it decreased the range of voltages that evoked tremor suppression without side effects. ${ }^{18}$

\section{CELLULAR EFFECTS OF DBS}

Common theories regarding the mechanisms of action of DBS focus on whether DBS excites or inhibits neurons in the target nucleus. Because the motor effects of high-frequency DBS in the STN or ventral intermediate nucleus of the thalamus resemble those seen following lesions of the target nucleus, early explanations of DBS function assumed that DBS inhibited or blocked the output of the target nucleus.

Indeed, the cell bodies (somas) of neurons in the stimulated nucleus are inhibited by high frequency DBS. ${ }^{19-22}$ In PD, STN neuron firing rates decreased from a baseline of $40 \mathrm{~Hz}$ to $7 \mathrm{~Hz}$ during STN DBS at $140 \mathrm{~Hz},{ }^{19}$ and microstimulation of the internal segment of globus pallidus (GPi) inhibited activity in GPi neurons near the electrode. ${ }^{21}$ A similar decrease in STN neuron firing rates was observed during STN high-frequency stimulation (HFS) of Parkinsonian monkeys. ${ }^{22}$ Such somatic inhibition may occur by either activation of the axons of inhibitory afferents, ${ }^{23}$ presynaptic depression at excitatory afferent axon terminals, ${ }^{24}$ and/or depolarization-induced blockade of somatic ion channels. ${ }^{25}$

On the other hand, there is abundant evidence that suggests that the efferent axons of the stimulated nucleus are activated by high frequency DBS. HFS in the internal segment of GPi of healthy monkeys reduced the firing rate of $77 \%$ of recorded thalamic cells, an inhibitory 


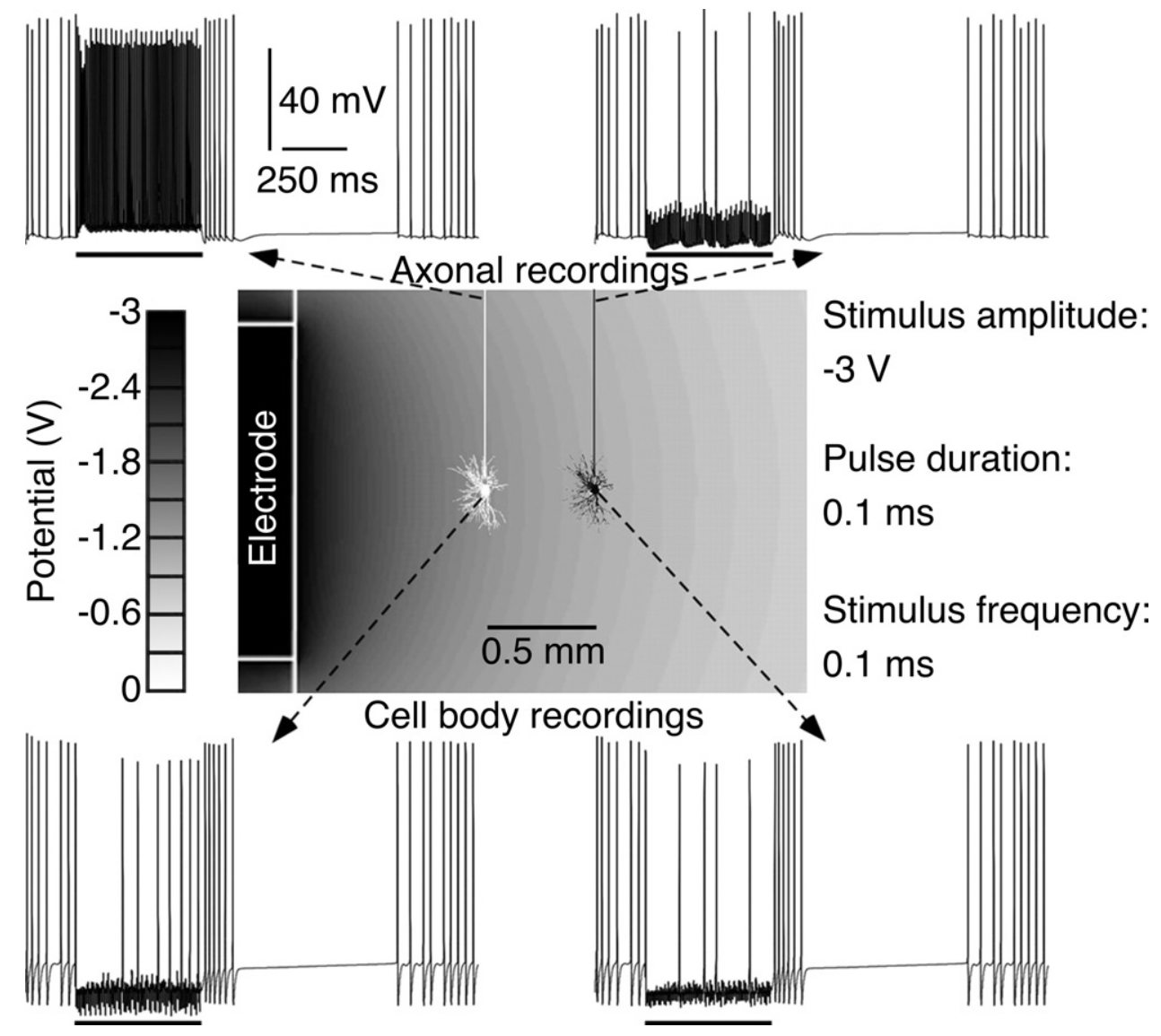

FIG 2. Simulations of the response of model thalamocortical relay neurons to deep brain stimulation show the dichotomous inhibition of cell bodies and excitation of axons. Cell bodies near the stimulating electrode (white neuron) are synaptically inhibited by activation of inhibitory afferent fibers. Axons of the same neurons (white) are excited directly by the stimulus. Cells whose axons are far enough from the electrode to prevent direct excitation (black neuron) are inhibited at the cell body, and this inhibition is reflected in the axon as well. Stimulation at $150 \mathrm{~Hz}$ was presented for $500 \mathrm{~ms}$ and is indicated by black bars below the transmembrane potential traces. Adapted with permission from Mclntyre et al. ${ }^{23}$

target of GPi axons, suggesting activation of GPi efferent fibers. ${ }^{26}$ HFS in the STN of Parkinsonian monkeys increased the firing rates in the external segment of globus pallidus (GPe) and GPi, excitatory targets of the STN, suggesting activation of STN efferent fibers. ${ }^{27}$ Positron emission tomography studies have also shown increased activity in the ipsilateral globus pallidus (GP) during STN DBS in humans with PD. ${ }^{28,29}$ Unilateral 6-hydroxydopamine lesions in rats increased glutamate and gamma-aminobutyric acid (GABA) in substantia nigra pars reticulata $(\mathrm{SNr})$ and $\mathrm{GP}$ as compared with intact rats, and these levels did not decrease during STN HFS, as they would have if DBS caused inhibition. ${ }^{30}$

These results appear contradictory. How can a neuron be excited and inhibited at the same time? These apparently conflicting experimental results were resolved with computational modeling indicating that DBS both inhibits and excites the target nucleus. ${ }^{5}$ During extracellular stimulation, action potential initiation occurs in the axon. ${ }^{31,32}$ While the cell bodies of neurons in the stimulated nucleus may indeed be inhibited, the axons of the same neurons are simultaneously excited (FIG. 2). ${ }^{23}$ The probability that microelectrodes in the stimulated nucleus record axonal activity is very low; therefore, recordings in the stimulated nucleus appropriately revealed only somatic inhibition, ${ }^{12}$ whereas recordings in downstream nuclei revealed axonal excitation. ${ }^{27}$

\section{Increased efferent activity from stimulated nucleus is propagated to downstream nuclei}

A question that arises from these results is whether the increased activity in the efferent axons of the stimulated nucleus is propagated via synaptic transmission to downstream nuclei. One hypothesis is that during HFS, changes take place in the somas of neurons in the stimulated nucleus that render these neurons incapable of releasing neurotransmitter, despite action potential propagation down the axons. ${ }^{33}$ Results that support this hypothesis include reduced secretions of prolactin, dopamine and norepinephrine in cells after 24 hours of HFS, suggesting that HFS suppresses the cellular machinery necessary to transmit activity to downstream neurons. ${ }^{34}$ 
However, this study was conducted in non-neuron cell types, and the application of these results to electrically active neurons is debatable. An alternative hypothesis is that repeated high-frequency activation leads to transmitter depletion and cessation of transmission from presynaptic to postsynaptic neurons. ${ }^{35}$

On the other hand, multiple studies confirm that the increased activity in efferent axons of the stimulated nucleus is propagated to downstream nuclei, although the propagation of DBS-induced action potentials may not be $1: 1 .^{36}$ During HFS in the STN, firing rates increased in the GPe and GPi (excitatory targets of the STN), which suggests that the efferent activation of the STN axons was propagated to downstream nuclei, and these effects were maintained for at least 5 minutes. ${ }^{27}$ Similarly, firing rates in the $\mathrm{SNr}$ of rats with neurolepticinduced catalepsy, which receives excitatory inputs from the STN, increased from $\sim 29 \mathrm{~Hz}$ to $\sim 42 \mathrm{~Hz}$ during HFS. ${ }^{37}$ Functional magnetic resonance imaging revealed that primary areas of activation during STN DBS were the ipsilateral thalamus and $\mathrm{GP},{ }^{38}$ and more specifically the $\mathrm{GPe}^{39}$, which are all downstream targets of the STN. Furthermore, glutamate levels in healthy rats increased in $\mathrm{GP}$ and $\mathrm{SNr}$ as a function of STN DBS frequency up to a plateau at $130 \mathrm{~Hz}^{40}$ The increase in glutamate levels was maintained during DBS at frequencies as high as $350 \mathrm{~Hz}$, but was slightly lower than at $130 \mathrm{~Hz}$ DBS, indicating that STN neurons released additional glutamate during high-frequency DBS. These data indicate that stimulated activity in the efferent axons of the stimulated nucleus is indeed propagated to downstream nuclei.

\section{NEURONAL FIRING PATTERNS IN MOVEMENT DISORDERS AND DURING DBS}

As compared with hypotheses regarding simple inhibition or excitation by DBS, currently available data indicate that effective DBS overrides pathological neuronal firing patterns present in movement disorders (such as bursts, low frequency oscillations, and synchronization) and replaces them with still abnormal, but apparently less deleterious patterns of activity. Although it is likely that the specific mechanism(s) by which DBS exerts its effects varies between diseases and target nuclei, the changes in neuronal activity during DBS appear to be ubiquitous.

\section{Basal ganglia and thalamic pathophysiology in movement disorders}

Complex changes in the rate, patterns, and information flow in neurons and networks of the basal ganglia and thalamus accompany movement disorders. ${ }^{41}$ There is an increase in the presence of bursts, low frequency oscillations, and synchronization in neurons in the basal gan- glia and thalamus in movement disorders..$^{22,42-48}$ Such changes in discharge patterns and synchronization play an important role in the pathophysiology of movement disorders. Motor symptoms including tremor, bradykinesia, and rigidity are associated with increased neuronal synchronization and low-frequency rhythmic oscillation, ${ }^{49-51}$ and bursts and oscillations parallel the appearance of motor symptoms in PD. ${ }^{52-54}$

A comparison of single unit GPi activity in subjects with PD versus Huntington's disease (HD) highlights the importance of the patterns of neuronal activity in addition to the rate of neuronal activity in movement disorders. The classical rate model of the basal ganglia divides the basal ganglia into the direct and indirect pathways (i.e., activity in the direct pathway initiates movements while activity in the indirect pathway inhibits movements). The clinical manifestations of PD and HD are polar opposites; PD is a hypokinetic disorder, whereas HD is a hyperkinetic disorder. Thus, the classical rate model predicts increases in the rates of GPi neuronal firing in PD (due to over-activity in the indirect pathway) and predicts decreases in the rates of GPi neuronal firing in HD. ${ }^{55,56}$ However, although the firing rates in the GPi are not significantly different in these two diseases, the firing patterns are different. ${ }^{47}$ The number of GPi neurons that participate in bursts is lower in HD subjects than in PD subjects. ${ }^{47}$ The burst indices and coefficients of variation of neuronal firing are also significantly lower in HD subjects than PD subjects. ${ }^{47}$ Finally, there is an increase in oscillatory activity in tremulous PD subjects as compared to HD subjects. ${ }^{47}$ Hence, there is an increase in bursting, irregular activity, and oscillatory firing patterns in the GPi of PD subjects as compared to HD subjects. $^{47}$

In addition to these findings in humans, in vivo recordings in nonhuman primates revealed that GPi firing rates are not significantly different between healthy and Parkinsonian (1-methyl-4-phenyl-1,2,3,6-tetrahydropyridine [MPTP]-treated) monkeys. ${ }^{52}$ These findings indicate that the symptoms of movement disorders are paralleled not only by changes in the rate of activity in the basal ganglia and thalamus, but perhaps more importantly by changes in the firing patterns of neurons. ${ }^{57}$

\section{Changes in neuronal firing during DBS}

DBS can cause changes in the patterns of activity of neurons surrounding the electrode. ${ }^{5}$ Changes in neuronal activity in the basal ganglia and thalamus during DBS include changes in rates and patterns of firing (i.e., pathological oscillations, bursting, and regularity of neuronal interspike intervals), as well as changes in metabolic rates, all of which are strongly dependent on the frequency of stimulation. The methods of assessing these changes in activity include recording single unit activity, recording local field potentials, simulating neuronal ac- 
tivity in computational models, measuring changes in metabolic activity, and functional imaging. Both single unit and local field potential recordings during DBS share the problem of contamination by the stimulation artifact. Although methods exist for subtracting or rejecting stimulus artifacts, these methods undoubtedly neglect some amount of activity and we do not know how much information is lost or the relative importance of the lost information. The technical difficulty of stimulating and recording from the same DBS lead, in conjunction with simultaneous artifact removal, previously precluded the recording of local field potentials in the stimulated nucleus during DBS in humans. However, methods to overcome the technical challenges of recording local field potentials during DBS were recently introduced, ${ }^{58}$ and studies using these methods are likely to be forthcoming. Computational models provide detailed information regarding the activity of neurons during simulated DBS, but are limited in their application by the assumptions made in generating the models and the uncertainty of model parameters. Finally, indirect methods of assessing neuronal activity (e.g., functional imaging and measurements of metabolic activity) are compatible with simultaneous DBS, but the link of these signals to neuronal activity is unclear, and they lack the ability to detect the fine structure of rates or patterns of neuronal activity. Despite the weaknesses of the individual techniques for assessing neuronal activity during DBS, evidence from multiple modalities indicates that DBS generates important changes in neuronal firing rates and patterns.

First, high-frequency stimulation reduces the pathological oscillations that are otherwise elevated in PD and other neurological disorders. ${ }^{59}$ High-frequency $(>70$ $\mathrm{Hz})$ STN DBS reduced pallidal beta oscillations (11-30 $\mathrm{Hz}$ ) in PD subjects in a manner similar to the administration of dopamine precursors, whereas $25 \mathrm{~Hz}$ DBS increased the power of pallidal beta oscillations. ${ }^{60} \mathrm{In}$ addition, high-frequency STN DBS at $130 \mathrm{~Hz}$ or $185 \mathrm{~Hz}$ attenuated STN beta oscillations in subjects with PD during the period just after DBS was turned off. ${ }^{20,61}$ Similar trends were observed in MPTP monkeys, where single unit activity can be recorded during HFS of the STN. The number of individual oscillatory STN neurons decreased from $37 \%$ just before HFS to $12 \%$ during HFS, and then returned to $35 \%$ within 3 minutes after HFS. $^{22}$

High-frequency DBS also generally decreases pathological synchronized burst activity in the basal ganglia and thalamus. The percent of $\mathrm{SNr}$ cells contributing to burst activity increased from $60 \%$ to $90 \%$ after neuroleptic injections in rats, and then decreased back to $62 \%$ during high-frequency STN stimulation. ${ }^{37}$ Similarly, the frequency and duration of thalamic bursts were reduced in response to HFS of the GPi in monkeys. ${ }^{26}$ Simulations of model thalamocortical relay neurons during $65 \mathrm{~Hz}$ and
$130 \mathrm{~Hz}$ stimulation also indicate that intrinsic bursts were disrupted more by $130 \mathrm{~Hz}$ DBS than by $65 \mathrm{~Hz}$ DBS. ${ }^{62}$ However, single-unit recordings in the STN of PD subjects suggest that the cell bodies in the STN may respond with more bursts during STN DBS. ${ }^{19}$ Four out of 21 neurons switched from an irregular firing pattern before DBS to a burst firing pattern during $140 \mathrm{~Hz}$ DBS, and one neuron switched from a burst pattern to an irregular pattern. Rat STN slice experiments demonstrated that STN HFS produced stable bursting activity in some STN neurons with intraburst interspike intervals that were phase-locked to the stimulation frequency or its subharmonics (e.g., one spike every 2 or 3 stimuli). ${ }^{63}$ In this case, it appears that STN neurons that burst during HFS do so in a regular fashion. However, the dichotomous nature of somatic and axonal firing may prevent the propagation of somatic bursts to the axon terminals. In fact, computational results demonstrated that antidromic invasion of action potentials created by phaselocked high frequency activation of axons can induce somatic bursts. ${ }^{64}$

Computational and experimental results suggest that neuronal firing becomes more regular during high-frequency DBS as compared with low frequency DBS or stimulation off. The coefficient of variation $(\mathrm{CV})$ of model neuron instantaneous firing rates increased during stimulation at frequencies $<50 \mathrm{~Hz}$, but decreased during stimulation at frequencies $>90 \mathrm{~Hz}$ (FIG. 1B) ${ }^{16}$ During HFS of the STN in rats with neuroleptic-induced catalepsy, the spontaneous firing rates of a portion of $\mathrm{SNr}$ neurons increased, whereas the rates of others decreased. However, "in all of the cases, the $\mathrm{SNr}$ firing pattern was regularized." 37 In addition, the entropy of interspike intervals (i.e., a measure of the irregularity or disorder of neuronal spiking) is decreased in GPi and GPe during HFS of the STN in MPTP monkeys, but increased by low-frequency stimulation. ${ }^{65}$ During low-frequency stimulation in these monkeys, interspike interval distributions returned to the pre-stimulation state around 10 ms after stimulus pulses, which demonstrated a failure to override pathological activity with low-frequency DBS. ${ }^{27}$ However, high-frequency STN DBS produced a four-phased orderly pattern of inhibition-excitationinhibition-excitation in the pallidum and thalamus that occurred in the $\sim 9 \mathrm{~ms}$ following stimulus pulses in the STN. ${ }^{27}$ Similar experiments performed during HFS in the GPi of MPTP monkeys also demonstrated that the intrinsic firing pattern in these neurons was replaced by "predicted, orderly discharge that was locked to the stimulus." 66 These results suggest that effective high-frequency DBS overrides pathological bursts and oscillations in the basal ganglia and thalamus, and replaces these deleterious patterns of activity with regularized firing that is phase-locked to the stimulus.

Additional support for the hypothesis that changes in 

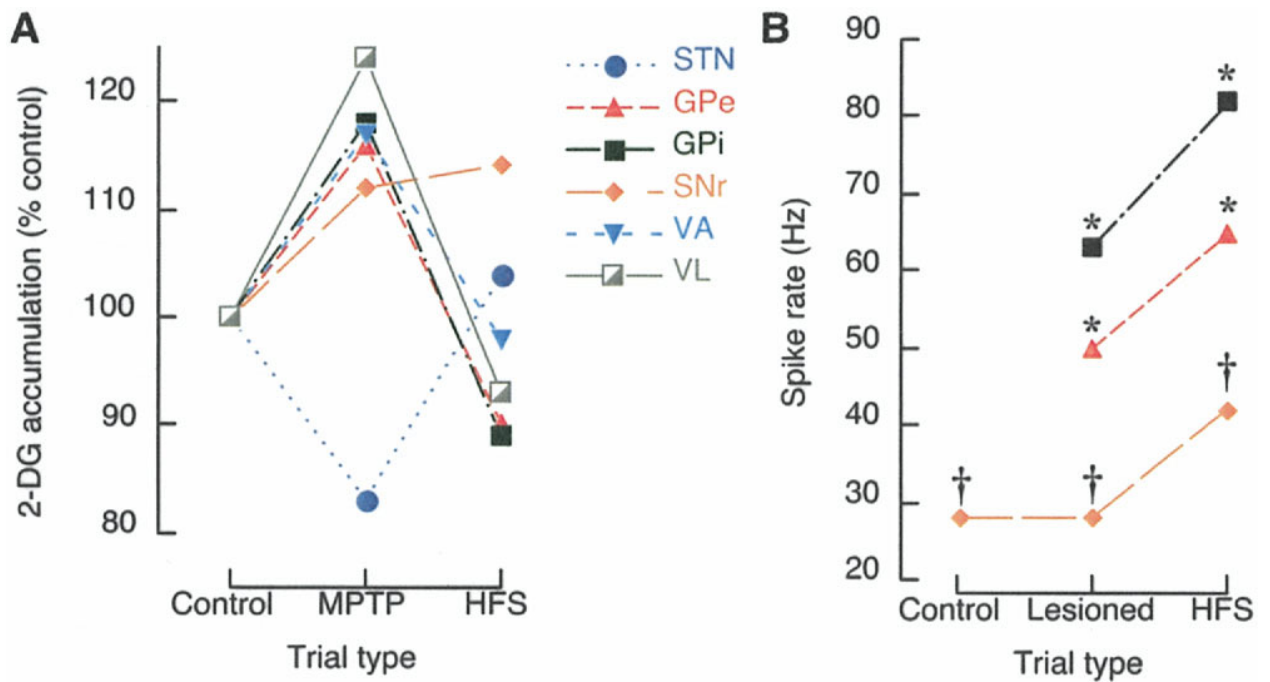

FIG 3. 2-deoxyglucose (2-DG) accumulation and firing rates in healthy monkeys, 1-methyl-4-phenyl-1,2,3,6-tetrahydropyridine (MPTP) monkeys, and MPTP monkeys after chronic high-frequency stimulation (HFS). A: Mean 2-DG accumulation in the basal ganglia and thalamus of healthy monkeys, MPTP monkeys, and MPTP plus subthalamic nucleus (STN) HFS monkeys, expressed as percent of control. ${ }^{69}$ Measurements provide an estimate of global synaptic activity in the relevant nuclei. After MPTP treatment, 2-DG accumulation increased in all nuclei except the STN where accumulation decreased. During STN HFS, glucose uptake levels returned close to normal. VA, ventral anterior thalamus; VL, ventral lateral thalamus. B: Mean spike rates in the external segment of globus pallidus (GPe) and internal segment of globus pallidus (GPi) in MPTP monkeys before and during HFS, as well as spike rates in substantia nigra pars reticulate $(\mathrm{SNr})$ of healthy rats, and rats with catalepsy before and during HFS. Legend in (A) applies to (B) as well. *Data from an individual monkey in Hashimoto, et al. ${ }^{27}$ †Data from Degos et al. ${ }^{37}$

the pattern of neuronal activity during DBS are more important than changes in the rate of activity is found in the opposite effects of GPe lesions and GPe DBS. Lesions in the GPe of MPTP monkeys worsened motor symptoms; retrieval times in a feeding task were longer than retrieval times prior to the GPe lesion, and total cage movement times decreased. ${ }^{67}$ Conversely, DBS in the GPe of subjects with PD ameliorated motor symptoms. ${ }^{68}$ These findings demonstrated that DBS does not act with the same mechanism as lesions (i.e., silencing neurons in the stimulated nucleus). Rather, they suggest that GPe DBS (as with DBS in other targets) overrides pathological activity in the stimulated nucleus.

Changes in metabolic rates of neurons in the basal ganglia during DBS also suggest that DBS alters neuronal firing patterns. Meissner et al. ${ }^{69}$ measured 2-deoxyglucose (2-DG) accumulation in healthy monkeys, MPTP monkeys, and MPTP monkeys after 10 days of STN HFS (FIG. 3A). All nuclei except the STN showed increased 2-DG accumulation in MPTP monkeys as compared with healthy monkeys. After 10 days of STN HFS, 2-DG accumulation was much closer to that of healthy monkeys than that of MPTP monkeys, suggesting some degree of normalization of activity in the basal ganglia and thalamus during STN HFS. Because 2-DG accumulation is a measure of global synaptic activity, it is intuitive to assume that the spike rates in nuclei with excitatory inputs from the STN would parallel 2-DG accumulation. However, this was not the case (see FIG. $3 \mathrm{~B}$ ); the mean firing rates of GPe and GPi neurons in
MPTP monkeys were higher during STN HFS than before STN HFS, ${ }^{27}$ and the mean firing rates of SNr neurons increased during STN HFS in rats with neurolepticinduced catalepsy. ${ }^{37}$ These results suggest that HFS drives seemingly opposite changes in synaptic activity and firing rates in the basal ganglia, which is a difference that may be exacerbated by differences in the duration of HFS in the previously described experiments. The divergence in 2-DG accumulation and firing rate trends with and without high frequency DBS may indicate that 2-DG accumulation depends not only on the firing rate of neurons and synapses in the nucleus of interest, but also on complex changes in the patterns of activity that take place in the same nucleus. Hence, changes in the patterns of activity with DBS may be more important than changes in the rate of activity with DBS.

\section{PARALLELS BETWEEN CHANGES IN FIRING PATTERNS AND MOTOR IMPROVEMENTS WITH DBS}

The results discussed in this review indicate that the effects of DBS are not mediated simply by excitation or inhibition of neuronal elements, but rather involve changes in the patterns of neuronal activity. High-frequency DBS overrides pathological activity in the stimulated nucleus and replaces the activity with regularized firing in the efferent axons of the stimulated nucleus. Both motor symptoms and neuronal firing patterns are strongly dependent on DBS frequency. Low frequencies 
either exacerbate or fail to improve motor symptoms and fail to override pathological activity patterns in neurons in the basal ganglia and thalamus. ${ }^{16,70}$ Conversely, highfrequency DBS improves motor symptoms and regularizes activity patterns in neurons in the basal ganglia and thalamus. ${ }^{16,70}$ The strong parallels between the clinical motor responses and patterns of neuronal responses to changes in DBS frequency suggest that the changes in neuronal activity underlie the observed changes in clinical motor symptoms.

Additional evidence for the link between regularization of neuronal activity and the clinical effects of DBS come from recent studies that examined the responses of both tremor and computationally modeled neurons to different stimulation paradigms (FIG. 4). ${ }^{16,18,62}$ As the frequency of stimulation increased, there was a decrease in both tremor and the $\mathrm{CV}$ of model neuron instantaneous firing rates, resulting in a strong correlation between model neuron CVs and tremor suppression $\left(\mathrm{R}^{2}=\right.$ 0.92; linear least squares fit) (FIG. 4A). Similar results were obtained in a more recent study that examined the changes in the regularity of neuronal firing and tremor suppression with changes in both the intensity and frequency of DBS. ${ }^{70}$ The amplitude- and frequency-dependent changes in the $\mathrm{CV}$ of neuronal firing closely paralleled the changes in tremor suppression with thalamic DBS. Further support for the link between regularization of neuronal activity and the clinical effects of DBS came from recent measurements of the effects of paired-pulse DBS on activity in bursting model thalamocortical neurons and on tremor in subjects with ET. ${ }^{62}$ More regular stimuli (i.e., those with small differences between the intrapair and interpair pulse intervals) were more effective at regularizing neuronal firing patterns and suppressing tremor. Correlations between the burst index of neuronal firing and tremor were again very strong across variations in the pulse-to-pulse frequency of stimulation $\left(\mathrm{R}^{2}=0.68\right.$; linear least squares fit) (FIG. 4B). ${ }^{62}$ In all of these cases, despite the fact that the simplified models did not include many of the features of the clinical experiments, there was an overarching correlation between the ability of stimulation to override intrinsic activity and its effectiveness at suppressing tremor.

\section{Hypotheses for ineffectiveness of low-frequency DBS}

Here, we present two possible explanations for why low-frequency DBS fails to alleviate motor symptoms and in some cases exacerbates symptoms. The long interpulse intervals present in low-frequency DBS trains may be too long to mask intrinsic activity in the stimulated nucleus, and even in the presence of stimulation, the intrinsic pathological activity persists. Under this hypothesis, low-frequency DBS fails to remove the deleterious patterns of activity in the stimulated nucleus, but

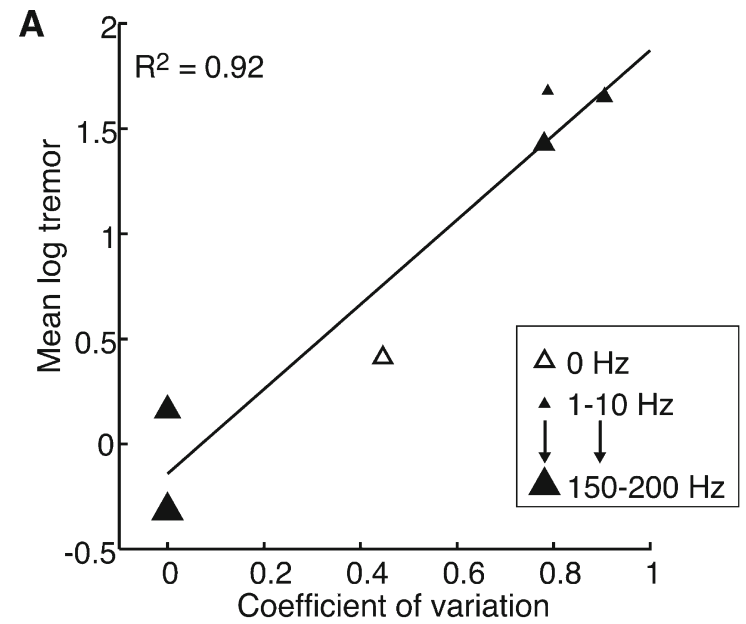

B

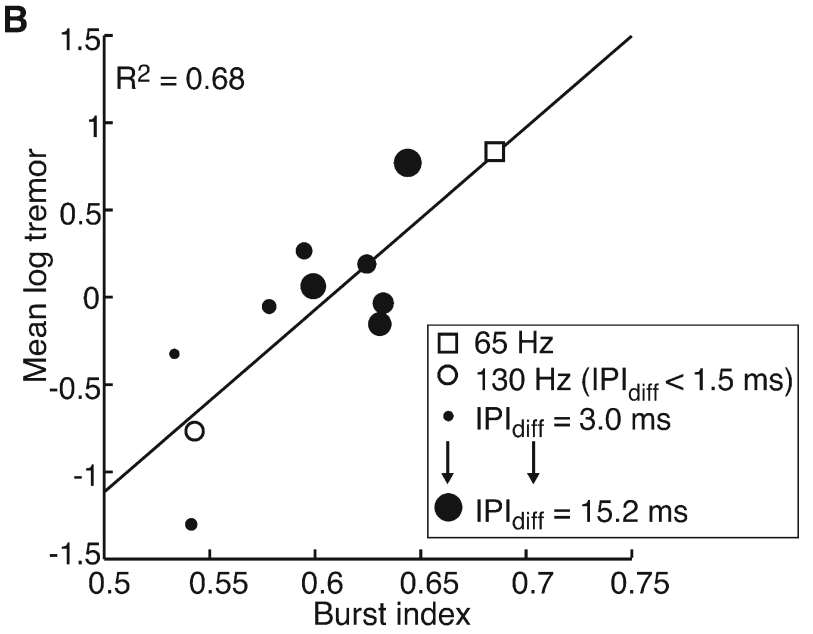

FIG 4. Tremor suppression in human subjects correlates with the ability of stimulation to override neuronal burst firing in model neurons. A: There is a positive correlation between log tremor power measurements and the coefficient of variation of instantaneous firing rates in a model thalamocortical neuron $\left(R^{2}=\right.$ $0.92){ }^{16,18}$ Both tremor and model neuron responses were recorded during stimulation at multiple frequencies (same data as in Figure 1). Each triangle represents the tremor power and coefficient of variation that correspond to a given stimulus frequency. The size of closed triangles increases with increasing frequency. B: There is likewise a positive correlation between the log tremor power measurements and the mean burst index across a population of 100 intrinsically bursting model thalamocortical neurons $\left(R^{2}=0.68\right){ }^{62}$ Tremor and model neuron firing patterns were measured during paired-pulse stimulation as a function of the difference between the interpair and intrapair pulse intervals $\left(\mathrm{IPI}_{\text {diff }}\right)$. Paired-pulse trains had an average rate of $130 \mathrm{~Hz}$. Measurements were also made during regular $130 \mathrm{~Hz}$ and $65 \mathrm{~Hz}$ stimulation. The burst index indicates the degree to which stimulation overrides pathological activity (i.e., an index of 1.0 indicates that there were no stimulus-induced changes to intrinsic activity and an index of 0.5 indicates that stimulationinduced activity completely replaced intrinsic activity). The size of the closed circles increases with increasing IPI ${ }_{\text {diff- }}$.

instead generates additional spikes on top of the intrinsic activity patterns. These additional spikes further disrupt the patterns of activity in the basal ganglia and thalamus, and through a yet to be defined mechanism, they lead to exacerbation of symptoms. It should be noted that such a 
change in the pattern of neuronal firing also increases the rate of firing in the stimulated nucleus, and the change in rate may affect symptom exacerbation in conjunction with the changes in pattern.

A second hypothesis is that the lack of effectiveness of low-frequency DBS results from the long interpulse intervals enabling and even promoting rebound bursts in the stimulated nucleus or in downstream nuclei. In other words, low-frequency DBS not only fails to override pathological activity, but actually produces and promotes synchronized pathological activity. Recent computation$\mathrm{al}^{71}$ and experimental ${ }^{72}$ results support this hypothesis. Person and Perkel ${ }^{72}$ measured the responses of songbird thalamic neurons to stimulation of inhibitory area $\mathrm{X}$ inputs (analogous to pallido-thalamic connections in humans). Area $\mathrm{X}$ fibers were stimulated with trains containing Poisson-distributed interpulse intervals with an average rate of $140 \mathrm{~Hz}$, and thalamic neurons responded with intermittent rebound spikes and bursts (FIG. 5A). The authors examined the timing of stimulus pulses preceding rebound events and found that in almost all cases, rebound spikes occurred after a pause between stimulus pulses of $20 \mathrm{~ms}$ or longer (FIG. 5B). During low-frequency DBS, pulses excite synchronously the synaptic inputs to the stimulated nucleus resulting in inhibition of the post-synaptic cells. ${ }^{73,74}$ Pauses in the input (i.e., the interpulse intervals) then enable rebound bursting in the stimulated nucleus, particularly in nuclei with robust inhibitory input. This sequence has also been observed in human thalamus just after microstimulation through the recording electrode. ${ }^{12}$ These bursts, enabled only when the interpulse interval is sufficiently long, can corrupt the fidelity of thalamic throughput ${ }^{75}$ and thereby exacerbate motor symptoms. Rebound bursts are also generated in STN slice neurons after pauses in high-frequency activation of $\mathrm{GABA}_{\mathrm{A}}{ }^{76}$ or $\mathrm{GABA}_{\mathrm{B}}{ }^{77}$ receptors, and these bursts may be propagated to basal ganglia outputs that in turn disrupt thalamic throughout.

\section{DBS mechanisms in diseases and targets where low- frequency DBS is effective}

The parallels between changes in neuronal firing patterns and motor symptoms as a function of frequency suggest that there is a minimum critical DBS frequency at which symptom alleviation and neuronal regularization occurs. ${ }^{70}$ However, DBS is effective in some nuclei and diseases at frequencies less than $90 \mathrm{~Hz}$. Early findings suggest that DBS at $25 \mathrm{~Hz}$ in the pedunculopontine nucleus (PPN) improves gait in PD. ${ }^{78}$ Case reports evaluating bilateral GPi DBS for HD yielded conflicting results during DBS at $40 \mathrm{~Hz}$; chorea was improved in one subject, ${ }^{79}$ but it was poorly controlled in another. ${ }^{80}$ Finally, studies examining the effects of the frequency of GPi DBS for subjects with dystonia yielded heterogeneous results. Stimulation at $50 \mathrm{~Hz}^{81}$ or $80 \mathrm{~Hz}^{82}$ im-
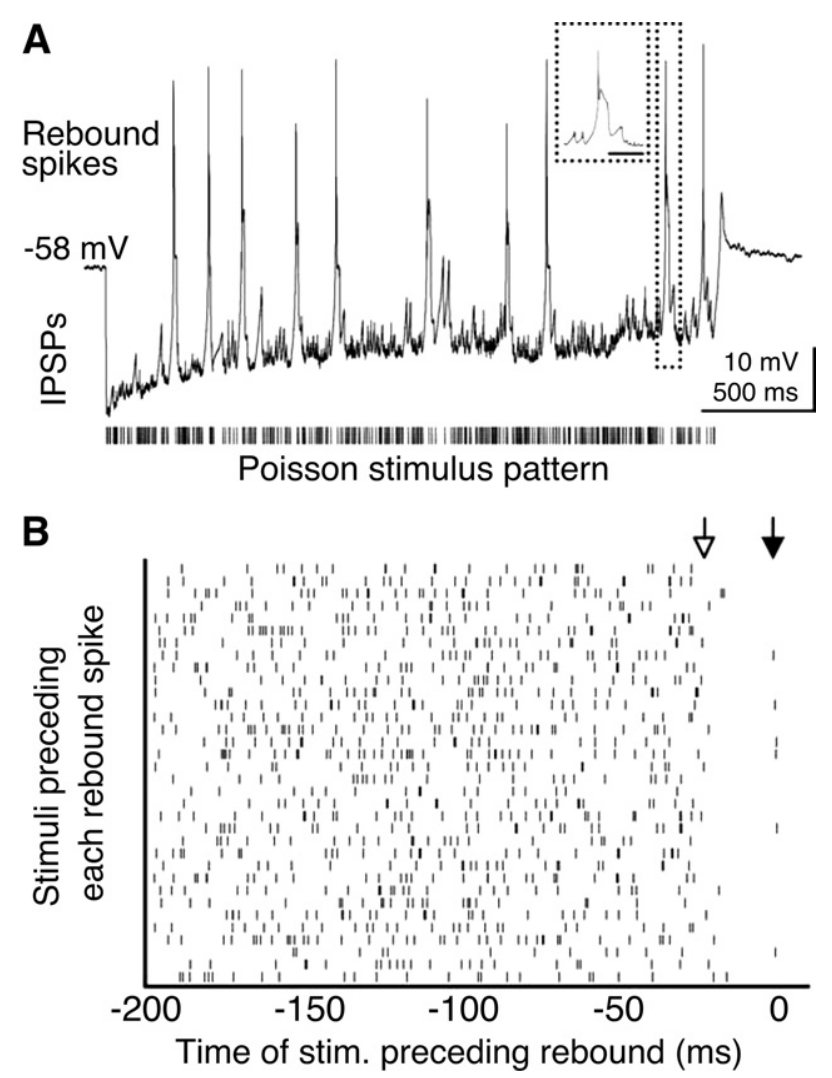

FIG 5. Inhibitory postsynaptic potentials drive rebound events in thalamic neurons during stimulation with high frequency Poisson trains. A: Perforated-patch recording of a thalamic neuron during delivery of Poisson distributed stimulation of area $\mathrm{X}$ afferents (mean rate of $140 \mathrm{~Hz}$ ). Tonic hyperpolarization is interrupted by occasional rebound events (inset; scale $=50 \mathrm{~ms}$ ). IPSPs, inhibitory postsynaptic potentials. B: Raster plot of stimulus pulses preceding rebound events. Each row represents the time that stimulus pulses were delivered during the $200 \mathrm{~ms}$ preceding a rebound event; there are 34 rebound events represented and each begins at the black arrow. A pause (i.e., interpulse interval) of 20 to $25 \mathrm{~ms}$ (beginning at the white arrow) immediately preceded burst responses in almost all cases. Reproduced with permission from Person and Perkel ${ }^{72}$ (Copyright, Elsevier Inc. [2005]).

proved Burke-Fahn-Marsden scale scores better in some subjects than stimulation at frequencies higher than 90 $\mathrm{Hz}$.

A simple, yet plausible, explanation for the effectiveness of low-frequency DBS in some targets and nuclei is that the pathological activity in these nuclei/diseases has a low intrinsic frequency, and thus can be overridden with DBS at lower stimulation frequencies (FIG. 6). ${ }^{16}$ That is not to say that high-frequency DBS will not work as well in these targets, but that the minimum effective frequency will be lower. First, while the effects of PPN DBS on PPN activity are unknown, firing rates in the PPN of a single PD subject (range, $8-29 \mathrm{~Hz}$ in 27 multiunit recordings) suggest the presence of low-rate intrinsic activity of the PPN in subjects with PD. ${ }^{83}$ Furthermore, cytochrome oxidase subunit I, choline acetyl transferase, and substance $\mathrm{P}$ mRNA expression de- 
A

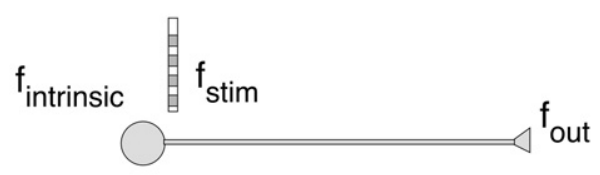

B

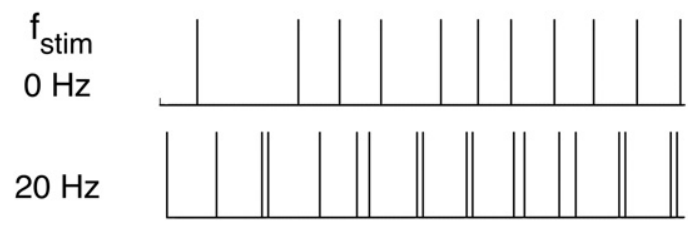

$100 \mathrm{~Hz}$

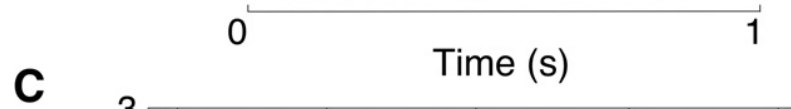

C

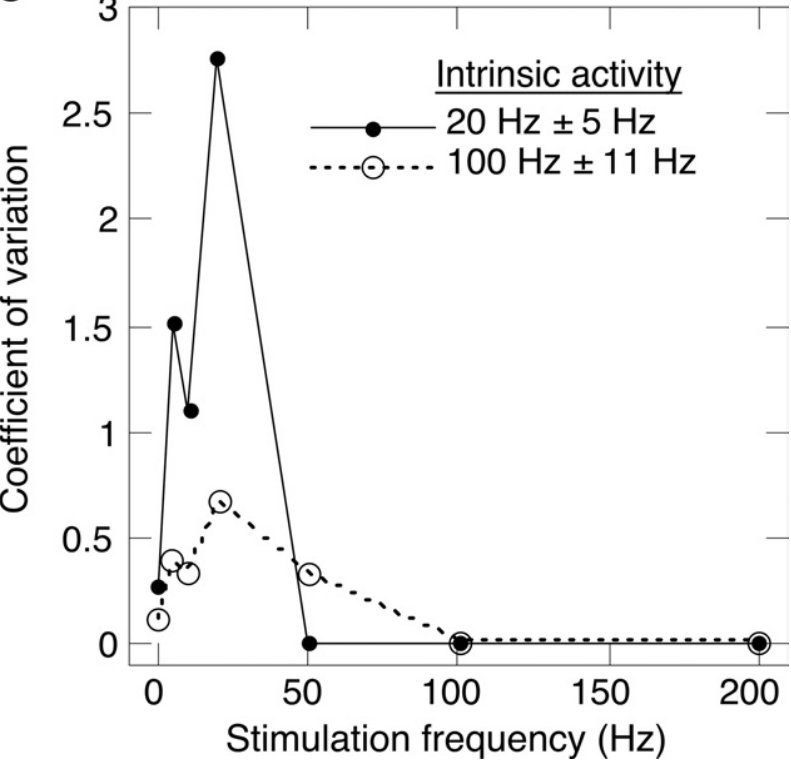

FIG 6. The minimum (critical) frequency needed to override intrinsic neuronal activity depends on the frequency of the intrinsic activity. A: An integrate-and-fire neuron was stimulated extracellularly, and the output of the axon was recorded. Intrinsic activity $\left(\mathrm{f}_{\text {intrinsic }}=\right.$ mean \pm standard deviation $\mathrm{Hz}$ ) was generated by an intracellular somatic current injection. B: The response of the integrate-and-fire neuron with intrinsic activity of $20 \pm 5 \mathrm{~Hz}$ to no stimulation $(0 \mathrm{~Hz})$ and to stimulation at $20 \mathrm{~Hz}$ and $100 \mathrm{~Hz}$. Stimulation at $20 \mathrm{~Hz}$ superimposes on top of the intrinsic activity, whereas stimulation at $100 \mathrm{~Hz}$ completely masks intrinsic activity and replaces it with activity locked to the stimulus train. C: Coefficient of variation of the instantaneous firing rate as a function of stimulus frequency for intrinsic frequencies of $20 \pm 5 \mathrm{~Hz}$ and $100 \pm 11 \mathrm{~Hz}$. Intrinsic activity at $20 \pm 5 \mathrm{~Hz}$ is masked (coefficient of variation $=0$ ) at a lower frequency than intrinsic activity at $100 \pm 11 \mathrm{~Hz}$. Adapted with permission from Grill et al. $^{16}$

creased significantly in the PPN of MPTP monkeys as compared with healthy monkeys, ${ }^{84}$ suggesting that the pathological activity in the PPN has a low intrinsic frequency. Second, although the average rate of GPi neuronal firing is approximately the same in PD and HD, (mean rate \pm standard error of the mean $=89.9 \pm 3 \mathrm{~Hz}$ in $\mathrm{PD}$ and $81.8 \pm 4.3 \mathrm{~Hz}$ in $\mathrm{HD}$ ), intrinsic neuronal activity in PD includes more bursting and irregular spiking. ${ }^{47}$ This difference in firing patterns may alter the response of the GPi to DBS and make it possible for GPi DBS to be effective in HD at a lower frequency than in PD. Synchronous burst activity includes many high-frequency spikes over short intervals during bursts (intraburst frequencies can be over $200 \mathrm{~Hz}$ ). To drive the cell out of bursting, a high-frequency stimulus may be necessary. However, results from $40 \mathrm{~Hz}$ GPi DBS in HD were conflicting, suggesting that the difference between the minimal effective frequencies for GPi DBS in PD and HD may not be very large. Third, the intrinsic firing rates of GPi neurons in a population of subjects with dystonia (i.e., heterogeneous etiologies) was much lower than in subjects with $\mathrm{PD}$ (mean rates \pm standard error of the mean $=95.2 \pm 2.3 \mathrm{~Hz}$ in PD and $55.3 \pm 1.3 \mathrm{~Hz}$ in dystonia). ${ }^{85}$ Similar findings were observed in subjects with cervical dystonia (mean rates \pm standard error of the mean $=91.7 \pm 3 \mathrm{~Hz}$ in $\mathrm{PD}$ and $71.4 \pm 2.2 \mathrm{~Hz}$ in cervical dystonia). ${ }^{86}$ Finally, simulations of the response of a model neuron to DBS demonstrate that the critical frequency (i.e., the minimum frequency required to mask intrinsic activity) increases as the frequency of intrinsic activity increases (FIG. 6) ${ }^{16}$ These results suggest that in disease states and nuclei where lower rates of activity accompany the pathology, low-frequency stimulation may provide effective relief of symptoms. Hence, the minimum frequency necessary for maximal symptom relief may vary across DBS targets and disease states.

\section{FUTURE PERSPECTIVES}

State-of-the-art implantable DBS systems have proven to be extremely useful in treating the motor symptoms of movement disorders; however, there is room for significant improvement in these devices. First, currently available DBS systems are open-loop stimulators. If the effectiveness of DBS is indeed tied to its ability to override pathological firing patterns and regularize neuronal firing, then it would be useful for DBS systems to have some feedback mechanism that measured such changes in neuronal firing. Second, DBS systems are limited by the heterogeneity of electrode placement and the inability of DBS leads to compensate for misplacement. Improved systems may include segmented electrodes to increase spatial selectivity. ${ }^{87}$ Finally, DBS systems can only produce periodic stimulus trains with constant interpulse intervals. If one of the mechanisms of DBS is to change the pattern of activity in the stimulated nucleus, then the temporal pattern of DBS may play an important role in the efficacy of DBS. These assertions are supported by a recent computational study wherein methods were outlined to identify stimulus patterns that effectively override pathological activity in PD, but at lower average frequencies. ${ }^{88}$ 


\section{CONCLUSIONS}

The motor symptoms of several movement disorders are treated effectively by DBS, and they show a strong dependence on the frequency of stimulation. We hypothesize that the changes in motor symptoms are related to patterns of activity in the stimulated nucleus. When the stimulus frequency is sufficiently high (i.e., above the critical frequency), DBS overrides intrinsic pathological activity in the target nucleus and replaces this activity with regularized neuronal firing. Although the resulting patterns of neuronal activity are certainly not normal (i.e., high-rate firing locked to the stimulus pulses), they are apparently less deleterious to motor function than the intrinsic, pathological patterns of activity present in movement disorders.

Acknowledgments: This work was supported by National Institutes of Health Grants R01NS040894 and R21NS055320, as well as a National Science Foundation Graduate Research Fellowship.

\section{REFERENCES}

1. Gross RE, Lozano AM. Advances in neurostimulation for movement disorders. Neurol Res 2000;22:247-258.

2. Krauss JK, Yianni J, Loher TJ, Aziz TZ. Deep brain stimulation for dystonia. J Clin Neurophysiol 2004;21:18-30.

3. Benabid AL, Pollak P, Gervason C, et al. Long-term suppression of tremor by chronic stimulation of the ventral intermediate thalamic nucleus. Lancet 1991;337:403-406.

4. McIntyre CC, Savasta M, Kerkerian-Le Goff L, Vitek JL. Uncovering the mechanism(s) of action of deep brain stimulation: activation, inhibition, or both. Clin Neurophysiol 2004;115:12391248.

5. Grill WM, McIntyre CC. Extracellular excitation of central neurons: implications for the mechanisms of deep brain stimulation. Thalamus Relat Syst 2001;1:269-277.

6. Kuncel AM, Grill WM. Selection of stimulus parameters for deep brain stimulation. Clin Neurophysiol 2004;115:2431-2441.

7. Benabid AL, Koudsie A, Benazzouz A, et al. Subthalamic stimulation for Parkinson's disease. Arch Med Res 2000;31:282-289.

8. Hodaie M, Wennberg RA, Dostrovsky JO, Lozano AM. Chronic anterior thalamus stimulation for intractable epilepsy. Epilepsia 2002;43:603-608.

9. Gross RE. Deep brain stimulation in the treatment of neurological and psychiatric disease. Expert Rev Neurother 2004;4:465-478.

10. Nuttin BJ, Gabriels L, van Kuyck K, Cosyns P. Electrical stimulation of the anterior limbs of the internal capsules in patients with severe obsessive-compulsive disorder: anecdotal reports. Neurosurg Clin N Am 2003;14:267-274.

11. Mayberg HS, Lozano AM, Voon V, et al. Deep brain stimulation for treatment-resistant depression. Neuron 2005;45:651-660.

12. Dostrovsky JO, Lozano AM. Mechanisms of deep brain stimulation. Mov Disord 2002;17(suppl 3):S63-68.

13. Moro E, Esselink RJ, Xie J, Hommel M, Benabid AL, Pollak P. The impact on Parkinson's disease of electrical parameter settings in STN stimulation. Neurology 2002;59:706-713.

14. Fogelson N, Kuhn AA, Silberstein P, et al. Frequency dependent effects of subthalamic nucleus stimulation in Parkinson's disease. Neurosci Lett 2005;382:5-9.

15. Timmermann L, Wojtecki L, Gross J, et al. Ten-Hertz stimulation of subthalamic nucleus deteriorates motor symptoms in Parkinson's disease. Mov Disord 2004;19:1328-1333.

16. Grill WM, Snyder AN, Miocinovic S. Deep brain stimulation creates an informational lesion of the stimulated nucleus. Neuroreport 2004;15:1137-1140.
17. Ushe M, Mink JW, Revilla FJ, et al. Effect of stimulation frequency on tremor suppression in essential tremor. Mov Disord 2004;19:1163-1168.

18. Kuncel AM, Cooper SE, Wolgamuth BR, et al. Clinical response to varying the stimulus parameters in deep brain stimulation for essential tremor. Mov Disord 2006;21:1920-1928.

19. Welter ML, Houeto JL, Bonnet AM, et al. Effects of high-frequency stimulation on subthalamic neuronal activity in parkinsonian patients. Arch Neurol 2004;61:89-96.

20. Foffani G, Ardolino G, Egidi M, Caputo E, Bossi B, Priori A. Subthalamic oscillatory activities at beta or higher frequency do not change after high-frequency DBS in Parkinson's disease. Brain Res Bull 2006;69:123-130.

21. Dostrovsky JO, Levy R, Wu JP, Hutchison WD, Tasker RR, Lozano AM. Microstimulation-induced inhibition of neuronal firing in human globus pallidus. J Neurophysiol 2000;84:570-574.

22. Meissner W, Leblois A, Hansel D, Bioulac B, Gross CE, Benazzouz A, et al. Subthalamic high frequency stimulation resets subthalamic firing and reduces abnormal oscillations. Brain 2005;128: 2372-2382.

23. McIntyre CC, Grill WM, Sherman DL, Thakor NV. Cellular effects of deep brain stimulation: model-based analysis of activation and inhibition. J Neurophysiol 2004;91:1457-1469.

24. Anderson TR, Hu B, Iremonger K, Kiss ZH. Selective attenuation of afferent synaptic transmission as a mechanism of thalamic deep brain stimulation-induced tremor arrest. J Neurosci 2006;26:841850.

25. Beurrier C, Bioulac B, Audin J, Hammond C. High-frequency stimulation produces a transient blockade of voltage-gated currents in subthalamic neurons. J Neurophysiol 2001;85:1351-1356.

26. Anderson ME, Postupna N, Ruffo M. Effects of high-frequency stimulation in the internal globus pallidus on the activity of thalamic neurons in the awake monkey. J Neurophysiol 2003;89: $1150-1160$.

27. Hashimoto T, Elder CM, Okun MS, Patrick SK, Vitek JL. Stimulation of the subthalamic nucleus changes the firing pattern of pallidal neurons. J Neurosci 2003;23:1916-1923.

28. Hershey T, Revilla FJ, Wernle AR, et al. Cortical and subcortical blood flow effects of subthalamic nucleus stimulation in PD. Neurology 2003;61:816-821.

29. Zhao YB, Sun BM, Li DY, Wang QS. Effects of bilateral subthalamic nucleus stimulation on resting-state cerebral glucose metabolism in advanced Parkinson's disease. Chin Med J (Engl) 2004; 117:1304-1308.

30. Windels F, Carcenac C, Poupard A, Savasta M. Pallidal origin of GABA release within the substantia nigra pars reticulata during high-frequency stimulation of the subthalamic nucleus. J Neurosci 2005;25:5079-5086.

31. Nowak LG, Bullier J. Axons, but not cell bodies, are activated by electrical stimulation in cortical gray matter. I. Evidence from chronaxie measurements. Exp Brain Res 1998;118:477-488.

32. Nowak LG, Bullier J. Axons, but not cell bodies, are activated by electrical stimulation in cortical gray matter. II. Evidence from selective inactivation of cell bodies and axon initial segments. Exp Brain Res 1998;118:489-500.

33. Benabid AL, Wallace B, Mitrofanis J, et al. A putative generalized model of the effects and mechanism of action of high frequency electrical stimulation of the central nervous system. Acta Neurol Belg 2005;105:149-157.

34. Xia R, Berger F, Piallat B, Benabid AL. Alteration of hormone and neurotransmitter production in cultured cells by high and low frequency electrical stimulation. Acta Neurochir (Wien) 2007;149: 67-73; discussion 73 .

35. Urbano FJ, Leznik E, Llinas RR. Cortical activation patterns evoked by afferent axons stimuli at different frequencies: an in vitro voltage-sensitive dye imaging study. Thalamus Relat Syst 2002;1:371.

36. Garcia L, Audin J, D’Alessandro G, Bioulac B, Hammond C. Dual effect of high-frequency stimulation on subthalamic neuron activity. J Neurosci 2003;23:8743-8751.

37. Degos B, Deniau J-M, Thierry A-M, Glowinski J, Pezard L, Maurice N. Neuroleptic-induced catalepsy: electrophysiological mechanisms 
of functional recovery induced by high-frequency stimulation of the subthalamic nucleus. J. Neurosci. 2005;25:7687-7696.

38. Jech R, Urgosik D, Tintera J, et al. Functional magnetic resonance imaging during deep brain stimulation: a pilot study in four patients with Parkinson's disease. Mov Disord 2001;16:1126-1132.

39. Phillips MD, Baker KB, Lowe MJ, et al. Parkinson disease: pattern of functional MR imaging activation during deep brain stimulation of subthalamic nucleus-initial experience. Radiology 2006;239: 209-216.

40. Windels F, Bruet N, Poupard A, Feuerstein C, Bertrand A, Savasta $\mathrm{M}$. Influence of the frequency parameter on extracellular glutamate and gamma-aminobutyric acid in substantia nigra and globus pallidus during electrical stimulation of subthalamic nucleus in rats. J Neurosci Res 2003;72:259-267.

41. Gale JT, Amirnovin R, Williams ZM, Flaherty AW, Eskandar EN. From symphony to cacophony: Pathophysiology of the human basal ganglia in Parkinson disease. Neurosci Biobehav Rev [Epub ahead of print] Apr 252007.

42. Bergman H, Wichmann T, Karmon B, DeLong MR. The primate subthalamic nucleus. II. Neuronal activity in the MPTP model of parkinsonism. J Neurophysiol 1994;72:507-520.

43. Hammond C, Bergman H, Brown P. Pathological synchronization in Parkinson's disease: networks, models and treatments. Trends Neurosci 2007;30:357-364.

44. Brown P, Oliviero A, Mazzone P, Insola A, Tonali P, Di Lazzaro V. Dopamine dependency of oscillations between subthalamic nucleus and pallidum in Parkinson's disease. J Neurosci 2001;21: 1033-1038.

45. Vitek JL, Chockkan V, Zhang JY, et al. Neuronal activity in the basal ganglia in patients with generalized dystonia and hemiballismus. Ann Neurol 1999;46:22-35.

46. Levy R, Ashby P, Hutchison WD, Lang AE, Lozano AM, Dostrovsky JO. Dependence of subthalamic nucleus oscillations on movement and dopamine in Parkinson's disease. Brain 2002;125: $1196-1209$

47. Tang JK, Moro E, Lozano AM, et al. Firing rates of pallidal neurons are similar in Huntington's and Parkinson's disease patients. Exp Brain Res 2005;166:230-236.

48. Nini A, Feingold A, Slovin H, Bergman H. Neurons in the globus pallidus do not show correlated activity in the normal monkey, but phase-locked oscillations appear in the MPTP model of parkinsonism. J Neurophysiol 1995;74:1800-1805.

49. Bergman H, Feingold A, Nini A, et al. Physiological aspects of information processing in the basal ganglia of normal and parkinsonian primates. Trends Neurosci 1998;21:32-38.

50. Deuschl G, Raethjen J, Lindemann M, Krack P. The pathophysiology of tremor. Muscle Nerve 2001;24:716-735.

51. Silberstein P, Oliviero A, Di Lazzaro V, Insola A, Mazzone P, Brown P. Oscillatory pallidal local field potential activity inversely correlates with limb dyskinesias in Parkinson's disease. Exp Neurol 2005;194:523-529.

52. Wichmann T, Bergman H, Starr PA, Subramanian T, Watts RL, DeLong MR. Comparison of MPTP-induced changes in spontaneous neuronal discharge in the internal pallidal segment and in the substantia nigra pars reticulata in primates. Exp Brain Res 1999; 125:397-409.

53. Amirnovin R, Williams ZM, Cosgrove GR, Eskandar EN. Visually guided movements suppress subthalamic oscillations in Parkinson's disease patients. J Neurosci 2004;24:11302-11306.

54. Magnin M, Morel A, Jeanmonod D. Single-unit analysis of the pallidum, thalamus and subthalamic nucleus in parkinsonian patients. Neuroscience 2000;96:549-564.

55. Albin RL, Young AB, Penney JB. The functional anatomy of basal ganglia disorders. Trends Neurosci 1989;12:366-375.

56. DeLong MR. Primate models of movement disorders of basal ganglia origin. Trends Neurosci 1990;13:281-285.

57. Wichmann T, DeLong MR. Pathophysiology of Parkinson's disease: the MPTP primate model of the human disorder. Ann N Y Acad Sci 2003;991:199-213.

58. Rossi L, Foffani G, Marceglia S, Bracchi F, Barbieri S, Priori A. An electronic device for artefact suppression in human local field potential recordings during deep brain stimulation. J Neural Eng 2007:4:96-106.
59. Llinas RR, Ribary U, Jeanmonod D, Kronberg E, Mitra PP. Thalamocortical dysrhythmia: A neurological and neuropsychiatric syndrome characterized by magnetoencephalography. Proc Natl Acad Sci U S A 1999;96:15222-15227.

60. Brown P, Mazzone P, Oliviero A, et al. Effects of stimulation of the subthalamic area on oscillatory pallidal activity in Parkinson's disease. Exp Neurol 2004;188:480-490.

61. Wingeier B, Tcheng T, Koop MM, Hill BC, Heit G, BronteStewart HM. Intra-operative STN DBS attenuates the prominent beta rhythm in the STN in Parkinson's disease. Exp Neurol 2006; 197:244-251.

62. Birdno MJ, Cooper SE, Rezai AR, Grill WM. Pulse-to-pulse changes in the frequency of deep brain stimulation affect tremor and modeled neuronal activity. J Neurophysiol 2007;98: $1675-1684$

63. Garcia L, D'Alessandro G, Fernagut PO, Bioulac B, Hammond C. Impact of high-frequency stimulation parameters on the pattern of discharge of subthalamic neurons. J Neurophysiol 2005;94:36623669.

64. Lee DC, Grill WM. Differential effects of electric field distribution on the activation of CNS neurons. Presented at the Annual Fall Meeting of the Biomedical Engineering Society; Program No. 915; October 16, 2004; Philadelphia, Pennsylvania.

65. Dorval AD, Russo GS, Hashimoto T, Xu W, Vitek JL, Grill WM. Subthalamic high-frequency stimulation regularizes pallidal and thalamic neural activity. Soc Neurosci 2005; Program No. 331.7.2005 (abstract).

66. Bar-Gad I, Elias S, Vaadia E, Bergman H. Complex locking rather than complete cessation of neuronal activity in the globus pallidus of a 1-methyl-4-phenyl-1,2,3,6-tetrahydropyridine-treated primate in response to pallidal microstimulation. J Neurosci 2004; 24:7410-7419.

67. Zhang J, Russo GS, Mewes K, Rye DB, Vitek JL. Lesions in monkey globus pallidus externus exacerbate parkinsonian symptoms. Exp Neurol 2006;199:446-453.

68. Vitek JL, Hashimoto T, Peoples J, DeLong MR, Bakay RA. Acute stimulation in the external segment of the globus pallidus improves parkinsonian motor signs. Mov Disord 2004;19:907-915.

69. Meissner W, Guigoni C, Cirilli L, et al. Impact of chronic subthalamic high-frequency stimulation on metabolic basal ganglia activity: a 2-deoxyglucose uptake and cytochrome oxidase mRNA study in a macaque model of Parkinson's disease. Eur J Neurosci 2007;25:1492-1500.

70. Kuncel AM, Cooper SE, Wolgamuth BR, Grill WM. Amplitudeand frequency-dependent changes in neuronal regularity parallel changes in tremor with thalamic deep brain stimulation. IEEE Trans Neural Syst Rehabil Eng 2007;15:190-197.

71. Babadi B. Bursting as an effective relay mode in a minimal thalamic model. J Comput Neurosci 2005;18:229-243.

72. Person AL, Perkel DJ. Unitary IPSPs drive precise thalamic spiking in a circuit required for learning. Neuron 2005;46:129-140.

73. Schlag J, Villablanca J. A quantitative study of temporal and spatial response patterns in a thalamic cell population electrically stimulated. Brain Res 1968;8:255-270.

74. McIntyre CC, Grill WM. Extracellular stimulation of central neurons: influence of stimulus waveform and frequency on neuronal output. J Neurophysiol 2002;88:1592-1604.

75. Rubin JE, Terman D. High frequency stimulation of the subthalamic nucleus eliminates pathological thalamic rhythmicity in a computational model. J Comput Neurosci 2004;16:211-235.

76. Bevan MD, Magill PJ, Hallworth NE, Bolam JP, Wilson CJ. Regulation of the timing and pattern of action potential generation in rat subthalamic neurons in vitro by GABA-A IPSPs. J Neurophysiol 2002;87:1348-1362.

77. Hallworth NE, Bevan MD. Globus pallidus neurons dynamically regulate the activity pattern of subthalamic nucleus neurons through the frequency-dependent activation of postsynaptic $\mathrm{GABA}_{\mathrm{A}}$ and $\mathrm{GABA}_{\mathrm{B}}$ receptors. J Neurosci 2005;25:6304-6315.

78. Stefani A, Lozano AM, Peppe A, et al. Bilateral deep brain stimulation of the pedunculopontine and subthalamic nuclei in severe Parkinson's disease. Brain 2007;130:1596-1607.

79. Moro E, Lang AE, Strafella AP, et al. Bilateral globus pallidus stimulation for Huntington's disease. Ann Neurol 2004;56:290-294. 
80. Hebb MO, Garcia R, Gaudet P, Mendez IM. Bilateral stimulation of the globus pallidus internus to treat choreathetosis in Huntington's disease: technical case report. Neurosurgery 2006;58:E383; discussion E383.

81. Alterman RL, Shils JL, Miravite J, Tagliati M. Lower stimulation frequency can enhance tolerability and efficacy of pallidal deep brain stimulation for dystonia. Mov Disord 2007;22:366-368.

82. Kumar R, Dagher A, Hutchison WD, Lang AE, Lozano AM. Globus pallidus deep brain stimulation for generalized dystonia: clinical and PET investigation. Neurology 1999;53:871-874.

83. Mazzone P, Lozano A, Stanzione P, et al. Implantation of human pedunculopontine nucleus: a safe and clinically relevant target in Parkinson's disease. Neuroreport 2005;16:1877-1881.

84. Gomez-Gallego M, Fernandez-Villalba E, Fernandez-Barreiro A, Herrero MT. Changes in the neuronal activity in the pedunculopontine nucleus in chronic MPTP-treated primates: an in situ hybridization study of cytochrome oxidase subunit I, choline acetyl transferase and substance P mRNA expression. J Neural Transm 2007;114:319-326.

85. Starr PA, Rau GM, Davis V, et al. Spontaneous pallidal neuronal activity in human dystonia: comparison with Parkinson's disease and normal macaque. J Neurophysiol 2005;93:3165-3176.

86. Tang JK, Moro E, Mahant N, et al. Neuronal firing rates and patterns in the globus pallidus internus of patients with cervical dystonia differ from those with Parkinson's disease. J Neurophysiol 2007;98:720-729.

87. Wei XF, Grill WM. Current density distributions, field distributions and impedance analysis of segmented deep brain stimulation electrodes. J Neural Eng 2005;2:139-147.

88. Feng XJ, Greenwald B, Rabitz H, Shea-Brown E, Kosut R. Toward closed-loop optimization of deep brain stimulation for Parkinson's disease: concepts and lessons from a computational model. J Neural Eng 2007;4:L14-21. 